\title{
EquiTime, le temps équitable
}

\section{Georges Weil}

\section{(2) OpenEdition}

Journals

Édition électronique

URL : https://journals.openedition.org/histoire-cnrs/9094

DOI : 10.4000/histoire-cnrs.9094

ISSN : 1955-2408

\section{Éditeur}

CNRS Éditions

Édition imprimée

Date de publication : 5 octobre 2009

ISSN : 1298-9800

Référence électronique

Georges Weil, "EquiTime, le temps équitable », La revue pour l'histoire du CNRS [En ligne], 24 | 2009, mis en ligne le 05 octobre 2009, consulté le 20 mai 2021. URL : http://journals.openedition.org/histoirecnrs/9094; DOl : https://doi.org/10.4000/histoire-cnrs.9094

Ce document a été généré automatiquement le 20 mai 2021.

Comité pour l'histoire du CNRS 


\title{
EquiTime, le temps équitable
}

\author{
Georges Weil
}

1 Beaucoup d'entreprises sont face à une problématique simple à formuler mais difficile à mettre en œuvre : comment construire, suivre, compter et analyser les plannings des collaborateurs tout en respectant les logiques des métiers?

L'oiseau fait son nid...

2 La recherche a débuté en 1990 à la suite de ma thèse ès sciences portant sur la gestion des rendez- vous multiples à l'hôpital et des plannings d'infirmières. Cette thématique a pris une plus-value sur le plan académique en 1995 avec une thèse soutenue par Kamel Heus et grâce à diverses publications dans des revues scientifiques. Malgré des débuts prometteurs, des clients potentiels et des encouragements à l'échelle locale voire nationale, aucune création d'entreprise ne se profilait à l'horizon. Or, il fallait bien franchir le pas pour ne pas "gâcher dix années de recherche ». Le transfert vers des industriels du secteur avait été tenté en faisant des visites croisées entre leurs bureaux d'étude et notre laboratoire. Sans succès. Soit ils n'envisageaient pas le marché et ses évolutions comme nous. Soit ils se considéraient comme plus rapides et plus performants. Nous ne l'avons su qu'après. Depuis, le marché nous a donné raison, et nous sommes devenus leurs concurrents. Certains ont disparu. D'autres se sont rapprochés pour tenter de nous racheter. La société a donc été incubée (même si le mot n'existait pas encore) avec l'appui du service de valorisation de l'université Joseph Fourier, dirigé par Denis Leroux, et de mon directeur de laboratoire, Jacques Demongeot. Les premières ventes en 1997 ont permis de tester les produits en clientèle et ont conduit aux constats suivants : si l'innovation était appréciée, les fonctionnalités basiques (non innovantes) avaient été négligées, et il fallait donc les développer. L'entreprise EquiTime a été créée dans le cadre de la loi sur l'innovation de 1999.

La journée de travail, un véritable marathon

3 Les travaux de recherche menés en laboratoire ont conduit à la mise en place de nouveaux paradigmes pour la planification et la gestion du temps. Chacun compte le temps en fonction de ses priorités. Il y a un temps vécu par les collaborateurs, un temps compté par l'entreprise (pour la paye). Ils ne sont pas nécessairement comparables. Par exemple, les mercredis sont payés à l'identique, mais ne sont pas des jours comme les 
autres pour les parents d'enfants scolarisés. Les outils puissants d'aide à l'élaboration des plannings aux cadres de proximité contribuent sensiblement à améliorer l'organisation: adéquation effectif et activité, qualité et productivité, satisfaction et fidélisation des collaborateurs. Ces résultats sont obtenus par l'association d'outils mathématiques d'optimisation combinatoire, d'interfaces homme/machine éprouvées par l'usage, et de méthodes de mise en oeuvre chez les clients. Les infirmières se sont révélées autant un modèle qu'un marché: simplicité d'usage pour des plannings complexes, nécessité d'une approche aide à la décision, plus qu'une approche de contrôle, notion d'équité dans l'établissement du planning.

Premiers clients, premiers marchés

4 Nous avons dû attendre 2002 pour travailler avec un centre hospitalier universitaire. Auparavant, nous avions gagné le marché de grands comptes hors santé tels EDF/RTE ou Flunch. Depuis, nous avons conquis le leadership dans les domaines de la santé et du médico-social et avons exploité d'autres secteurs variés avec des clients pour le moins prestigieux: la Comédie française, la société de la tour Eiffel, quatre sociétés d'autoroute dont Sanef, l'Imprimerie nationale, la Française des jeux, Médiamétrie, et bien d'autres. Cette approche de la recherche nous a permis d'aller au-delà du secteur médical et nous invite désormais à modéliser et repérer des leviers d'innovation réellement perçue comme telle par les clients. Cette recherche transdisciplinaire embrasse les mathématiques appliquées, l'informatique, les interfaces homme/ machine, mais aussi la sociologie des organisations.

Une aide académique efficace

5 Nous souhaitons rendre hommage à l'Anvar (aujourd'hui Oseo) qui nous a épaulés financièrement et juridiquement et à la filiale d'investissement du CNRS porteuse des actions détenues par l'université Joseph Fourier. L'une comme l'autre ont pris le risque de nous choisir alors que nous étions jeunes et inexpérimentés en matière de transfert de technologie. Sans doute avaient-elles compris que nous leur apportions ce qu'elles ne trouvaient pas ailleurs chez nos concurrents plus établis mais moins innovants. Les investisseurs et nos partenaires internationaux (Oracle et Microsoft par exemple) ont fait montre d'un intérêt toujours grandissant, amplifiant ainsi ma motivation d'entrepreneur et celle de certains de mes collaborateurs. Certains de nos clients et de leurs décideurs formés par la recherche, se sont déclarés prêts à prendre un risque calculé en nous faisant confiance. C'est le cas de Thierry Maroye, à l'époque directeur informatique d'Agapes, la maison mère des restaurants Flunch, Pizza Pai. Enfin, la recherche - mais également l'enseignement et la vulgarisation - et les qualités gagnées dans ce domaine, sont utiles à la fois pour la vente aux clients et pour la formation des collaborateurs. Elle conduit à un style de management qui a son intérêt, ses limites aussi, et qu'il convient d'équilibrer avec une approche classique de la gestion des hommes et d'une entreprise. 


\section{RÉSUMÉS}

Dans certaines entreprises où les régimes de travail sont complexes et où la planification des horaires est un imbroglio permanent, les responsables de l'élaboration et du suivi des plannings cherchent toujours à gagner du temps. Georges Weil nous présente EquiTime en cinq minutes chrono...

\section{AUTEUR}

\section{GEORGES WEIL}

Georges Weil, co-créateur d'EquiTime, est chercheur associé au laboratoire « Techniques de l'imagerie, de la modélisation et de la cognition » (TIMC). 